ESJ Humanities

\title{
Plateforme d'innovations technologiques et organisationnelles : Leçons apprises de l'étuvage du riz à Malanville (Bénin)
}

\section{Corneille Dassou}

Laboratoire de Sociologie et de Vulgarisation Rurales, Ecole d'Economie, de Socio-Anthropologie et de Communication pour le développement rural,

Faculté des Sciences Agronomiques, Abomey-Calavi, Bénin

\section{Espérance Zossou}

Laboratoire de Sociologie et de Vulgarisation Rurales, Ecole d'Economie, de

Socio-Anthropologie et de Communication pour le développement rural,

Faculté des Sciences Agronomiques, Abomey-Calavi, Bénin

Affiliation antérieure : Africa Rice Center, Cotonou, Bénin

\section{Alidou Assouma-Imorou \\ Simplice Vodouhe \\ Rigobert C. Tossou}

Laboratoire de Sociologie et de Vulgarisation Rurales, Ecole d'Economie, de Socio-Anthropologie et de Communication pour le développement rural,

Faculté des Sciences Agronomiques, Abomey-Calavi, Bénin

\section{Doi:10.19044/esj.2021.v17n24p71}

Submitted: 04 March 2021

Accepted: 02 July 2021

Published: 31 July 2021
Copyright 2021 Author(s)

Under Creative Commons BY-NC-ND

4.0 OPEN ACCESS

Cite As:

Dassou C., Zossou E., Assouma-Imorou A., Vodouhe S. \& Tossou R.C. (2021). Plateforme d'innovations technologiques et organisationnelles : Leçons apprises de l'étuvage du riz à Malanville (Bénin). European Scientific Journal, ESJ, 17(24), 71.

https://doi.org/10.19044/esj.2021.v17n24p71

\section{Résumé}

La plateforme d'innovation (PI) est une approche multi-acteurs pour des solutions aux problèmes complexes. Elle est d'actualité en Afrique Subsaharienne confrontée à de nombreux défis agricoles. Cette étude utilise une perspective systémique pour comprendre le processus de génération et de diffusion d'innovation relative à la post-récolte du riz local au Bénin. La collecte des données quantitatives et qualitatives a été faite avec des questionnaire et guides d'entretien auprès de 300 femmes étuveuses de riz, membres de la PI à Malanville, et sélectionnées de façon aléatoire et stratifiée. Les tests $t$ de Student et de Wilcoxon et le modèle de régression binomiale 
Logit ont été utilisés pour analyser les données. Les résultats montrent que l'introduction de la technologie améliorée d'étuvage de riz dans la PI a servi de catalyseur pour le développement d'un espace d'apprentissage et de changement caractérisé par : (i) la génération d'innovations technologiques ; (ii) l'organisation en groupements pour l'amélioration des pratiques et l'accès au micro-crédit et aux sources formelles d'apprentissage ; et (iii) la diffusion des innovations générées au sein de la PI. Cette étude confirme que les approches multi-acteurs favorisent l'apprentissage individuel et social, l'expérimentation et la modification des innovations, et le renforcement du capital social pour un développement agricole inclusif et durable.

Mots clés : Plateforme d'innovation, riz étuvé, diffusion, Bénin

\section{Innovation Platform as a space of technological and organisational innovations generation and diffusion: Lessons learnt from rice parboiling in Malanville (Benin)}

\section{Corneille Dassou}

Laboratoire de Sociologie et de Vulgarisation Rurales, Ecole d'Economie, de Socio-Anthropologie et de Communication pour le développement rural,

Faculté des Sciences Agronomiques, Abomey-Calavi, Bénin

\section{Espérance Zossou}

Laboratoire de Sociologie et de Vulgarisation Rurales, Ecole d'Economie, de

Socio-Anthropologie et de Communication pour le développement rural,

Faculté des Sciences Agronomiques, Abomey-Calavi, Bénin

Affiliation antérieure : Africa Rice Center, Cotonou, Bénin

\section{Alidou Assouma-Imorou \\ Simplice Vodouhe \\ Rigobert C. Tossou}

Laboratoire de Sociologie et de Vulgarisation Rurales, Ecole d'Economie, de

Socio-Anthropologie et de Communication pour le développement rural,

Faculté des Sciences Agronomiques, Abomey-Calavi, Bénin

\section{Abstract}

The Innovation Platform (IP) is a multi-stakeholder approach to address complex challenges. This is topical in Sub-Saharan Africa that is facing complex agricultural challenges. This study used a systems perspective to understand the process of generation and diffusion of innovation related to the postharvest of local rice in Benin. Quantitative and qualitative data were collected using questionnaires and interview guides with 300 women rice 
parboilers, randomly and stratified selected from the IP members in Malanville. Data were analyzed using the Student t-test, Wilcoxon test and the Logit binomial regression model. Results show that the introduction of the improved rice parboiling technology in the IP served as a catalyst for the development of a space for learning and change characterized by: (i) the generation of technological innovations; (ii) the organization into groups for the improvement of practices, access to micro-finance and to formal learning sources; and (iii) the dissemination of innovations generated within the IP. This study confirms that multi-stakeholder approaches promote individual and social learning, experimentation and modification of innovations, and strengthening of social capital for inclusive and sustainable agricultural development.

Keywords: Innovation platform, rice parboiling, dissemination, Benin

\section{Introduction}

En dépit de son urbanisation galopante, l'Afrique demeure un continent essentiellement rural à faible taux d'alphabétisation et une prédominance de petits exploitants agricoles. L'agriculture y tient donc une place centrale dans l'activité économique par environ $60 \%$ de la population active. De plus, l'agriculture constitue un secteur devant répondre à l'impérieuse nécessité d'innover afin de renforcer non seulement les performances économiques des exploitations, mais aussi leur efficacité sociale et environnementale (Pouch, 2015).

L'histoire de la vulgarisation agricole en Afrique Sub-Saharienne fait cas de l'innovation qui était perçue comme étant insufflée par la recherche avec l'utilisation d'approches dirigistes et paternalistes (Faure et al., 2018 ; Zossou et al., 2021). Les nombreuses critiques à l'encontre des approches dirigistes ainsi que les résultats mitigés obtenus, malgré l'importance des ressources consenties, ont conduit à des approches plus participatives et inclusives afin de mieux prendre en compte les besoins réels et concrets des communautés agricoles (MAEP, 2007 ; Zossou et al., 2020 ; Zossou et al., 2021).

Dans un tel contexte de changement de paradigme dans le processus de production et d'utilisation de l'innovation agricole, une attention particulière est de plus en plus mis sur le caractère multidimensionnel des innovations et la nécessité de construire des liens et des réseaux efficaces dans un processus évolutif (Shut al., 2016 : Zossou et al., 2020).

Les plateformes d'innovation et ou plateformes multi-acteurs, relevant d'une perspective systémique de l'innovation agricole, sont donc de plus en plus utilisées pour faire face aux innombrables défis auxquels fait face l'agriculture en Afrique Sub-Saharienne en général et au Bénin en particulier 
(Jeunier et al., 2000 ; Leeuwis, 2010 ; Homann-Kee Tui et al., 2013; Schut et al., 2016 ; Angbo-Kouakou et al., 2016). Les Plateformes d'Innovation (PI) sont des approches systémiques utilisées pour renforcer les trois piliers d'un système d'innovation à savoir l'apprentissage, les institutions et les interactions (Van Mele et al., 2010 ; Homann-Kee Tui et al., 2013; Nederlof et Pyburn, 2012 ; ILRI, 2013 ; Schut et al., 2016).

Les efforts de développement de la riziculture font face à de nombreux défis au Bénin et concerne principalement la qualité du produit final et sa valorisation sur le marché (Konnon et al., 2014 ; Houssou et al., 2016). Dans le but de relever les défis qui s'imposent à la post-récolte du riz étuvé local au Bénin, une plateforme d'innovation a été mise en place de façon participative et inclusive à Malanville avec l'appui financier et technique du Centre du Riz pour l'Afrique (AfricaRice). L'adoption d'une telle approche systémique dans la chaine de valeur du riz étuvé est susceptible de stimuler l'interaction entre les acteurs pour la production et l'utilisation d'innovation pouvant impacter positivement la compétitivité du riz local et améliorer les moyens d'existence des acteurs à la base. La présente étude se propose d'analyser la contribution de la plateforme d'innovation mise en place dans le renforcement des interactions entre les acteurs ; avec un regard sur la génération et la diffusion d'innovations technologiques et organisationnelles.

\section{Materiels et Methodes \\ Milieu d'étude}

Cette recherche a été réalisée au Bénin précisément dans la commune de Malanville qui est le bassin rizicole du pays. Cette commune s'étend entre $11^{\circ} 52^{\prime} 6 \mathrm{~N}$ de latitude et $3^{\circ} 23^{\prime} 23 \mathrm{E}$ de longitude. Elle est limitée au Nord par la République du Niger, au Sud par les communes de Kandi et de Ségbana, à l'Ouest par la commune de Karimama et à l'Est par la République Fédérale du Nigéria. Elle couvre une superficie de $3,016 \mathrm{~km}^{2}$ dont 8000 ha de terres cultivables (Ahoyo, 2006). Selon l'Institut National de la Statistique et de l'Analyse Economique (INSAE) en 2013, sa population totale est estimée à 168641 avec 49,62\% d'hommes et 50,38\% de femmes. La commune de Malanville compte cinq (5) arrondissements à savoir Garou, Guene, Madecali, Tomboutou et Malanville.

\section{Echantillonnage et Collecte de données}

La méthode d'échantillonnage aléatoire stratifié suivi d'un tirage aléatoire simple a été utilisée pour sélectionner 300 femmes étuveuses issues d'une population de 538 individus, inégalement réparties dans 22 groupements qui font partie de la plateforme d'innovation. A ce titre, un coefficient d'échantillonnage (Coef = Taille de l'échantillon / Population totale) a été calculé et multiplié à l'effectif total de chaque groupement. Ce qui a permis 
d'avoir l'effectif à échantillonner dans chaque groupement. Ensuite, un tirage aléatoire simple a été effectué avec la fonction «Alea » de Excel pour chaque groupement en fonction de l'effectif requit après l'application du quota.

La collecte de données a débuté par la revue documentaire sur le thème de référence. Ainsi, des rapports d'activités, articles scientifiques, mémoires et thèses universitaires ont été consultés pour circonscrire la problématique et définir les objectifs de l'étude. Ensuite, une enquête exploratoire a eu lieu pour recadrer les objectifs et tester les outils de collecte élaborés pour la circonstance. Par ailleurs, les données primaires ont été collectées au cours d'une enquête fine pour laquelle un questionnaire réalisé par le logiciel Sphinx a été utilisé.

\section{Traitement et analyse de données}

Quant aux traitements et analyses des données, la statistique descriptive a été utilisée à travers les paramètres de positionnement et les fréquences relatives. Aussi, le test de Wilcoxon, le test t de Student et la régression Logit ont-ils été utilisés pour réaliser des analyses plus approfondies.

Pour analyser l'amélioration des pratiques à chaque étape du processus d'étuvage du riz, des scores ont été soumis à l'appréciation des femmes étuveuses avant et après introduction de l'innovation «GEM». Ces scores varient de 0 à 10 où 0 signifie pas du tout d'amélioration et 10 la plus grande amélioration possible. Des moyennes ont été ensuite calculées et comparées par étape (avant et après GEM). Le test de Wilcoxon a permis de confirmer ou d'infirmer les différences observées.

\section{Resultats}

\section{Informations sociodémographiques des femmes étuveuses du riz}

Les femmes étuveuses ont une moyenne d'âge de 39 ans $( \pm 11,22)$ avec une ancienneté de 9 ans $( \pm 5,72)$ dans l'activité d'étuvage (Tableau 1). En outre, elles sont dans leur majorité mariées (99\%) avec un faible niveau d'instruction $(21,33 \%)$ et une taille moyenne de ménage égale à $7,08( \pm 2,46)$. Par ailleurs, toutes ont une appartenance à un groupement et exercent pour la plupart $(88,33 \%)$ l'étuvage comme activité principale. L'agriculture demeure l'activité secondaire pour $79 \%$ de ces femmes étuveuses. 
Tableau 1 : Définition des variables et profil sociodémographique des femmes étuveuses

\begin{tabular}{|c|c|c|c|}
\hline Variables & Description & Paramètre & $\begin{array}{l}\text { Répondants } \\
(\mathbf{n}=\mathbf{3 0 0})\end{array}$ \\
\hline Age & Age des répondants (en année) & $\begin{array}{l}\text { moyenne Ecart- } \\
\text { type }\end{array}$ & $39,35 \pm 11,22$ \\
\hline Expérience & $\begin{array}{l}\text { Nombre d'années d'expérience } \\
\text { dans l'activité d'étuvage du riz }\end{array}$ & $\begin{array}{l}\text { moyenne Ecart- } \\
\text { type }\end{array}$ & $9,15 \pm 6$ \\
\hline Taille du ménage & $\begin{array}{l}\text { Nombre de personnes vivant } \\
\text { dans le ménage du répondant }\end{array}$ & $\begin{array}{l}\text { moyenne Ecart- } \\
\text { type }\end{array}$ & $7,08 \pm 2,46$ \\
\hline \multirow[t]{5}{*}{ Niveau d'instruction } & Aucun & $\%$ de répondantes & 78,67 \\
\hline & Niveau primaire & $\%$ de répondantes & 7,33 \\
\hline & Premier cycle & $\%$ de répondantes & 3,67 \\
\hline & Second cycle & $\%$ de répondantes & 0,33 \\
\hline & Ecole coranique & $\%$ de répondantes & 10 \\
\hline \multirow[t]{3}{*}{ Situation matrimoniale } & Célibataire & $\%$ de répondantes & 0,33 \\
\hline & Mariée & $\%$ de répondantes & 99 \\
\hline & Veuve & $\%$ de répondantes & 0,67 \\
\hline \multirow{2}{*}{$\begin{array}{c}\text { Membres d'un } \\
\text { Groupement }\end{array}$} & Oui & $\%$ de répondantes & 1 \\
\hline & Non & $\%$ de répondantes & 0 \\
\hline \multirow[t]{4}{*}{ Activité principale } & Agriculture & $\%$ de répondantes & 9 \\
\hline & Etuvage & $\%$ de répondantes & 88,33 \\
\hline & Commerce & $\%$ de répondantes & 2 \\
\hline & Transformation agro-alimentaire & $\%$ de répondantes & 0,67 \\
\hline \multirow[t]{5}{*}{ Activité secondaire } & Agriculture & $\%$ de répondantes & 79 \\
\hline & Etuvage & $\%$ de répondantes & 12 \\
\hline & Commerce & $\%$ de répondantes & 8 \\
\hline & Artisanat & $\%$ de répondantes & 0,33 \\
\hline & Transformation agro-alimentaire & $\%$ de répondantes & 0,67 \\
\hline
\end{tabular}

Source : Données de terrain

Historique sur la mise en place de la Plateforme d'Innovation (PI) et la mise en place du système amélioré d'étuvage du riz « GEM »

La PI de la chaine de valeur du riz étuvé a été initiée de manière participative et inclusive à Malanville en Décembre 2015 dans le cadre du projet «Appui à la recherche pour le développement des cultures stratégiques en Afrique (SARD-SC)», financé par la Banque Africaine de Développement (BAD). Cette plateforme a été mise en place dans le but de servir de creuset pour l'identification, la validation, l'opérationnalisation, l'utilisation et le suivi des innovations appropriées identifiées par les acteurs eux-mêmes à la base. Les acteurs qui ont participé à la mise en place de la plateforme sont divers et catégorisés comme suit: semenciers, agriculteurs, Institutions de Micro-Finance (IMF), femmes étuveuses, meunières, courtiers, commerçants, agents de vulgarisation du Secteur Communal pour le Développement Agricole (SCDA), les chercheurs du Centre du Riz pour l'Afrique (AfricaRice). Le point d'entrée de la PI est le riz étuvé local de bonne qualité. Une équipe de facilitation et de coordination de la PI a été mise en place et 
composée des représentants de chacune des parties prenantes. Depuis sa création, la plateforme a bénéficié des appuis techniques et financiers d'AfricaRice dont l'installation et la formation à l'utilisation du « GEM », la mise en place d'équipements agricoles grâce à l'initiative d'urgence japonaise, la formation des jeunes à la mécanisation, l'initiation à un bon emballage et étiquetage du riz, et la formation sur RiceAdvice qui est un outil d'aide à la décision pour la gestion des cultures rizicoles.

Avant la mise en place du système amélioré d'étuvage du riz «GEM » à Malanville, les méthodes d'étuvage existantes dans ce milieu étaient traditionnelles et semi-moderne. La méthode traditionnelle se fait par le trempage du paddy dans de l'eau froide ou chaude pendant environ 12 heures à 72 heures dans une marmite en fonte d'aluminium. Le paddy égoutté après cette étape est ensuite précuit dans la même marmite avec une petite quantité d'eau. Avec une telle méthode, la précuisson du paddy n'est pas uniforme du sommet au fond de la marmite. Ce qui donne un produit hétérogène à la mouture avec un fort taux de brisure. Pour pallier à ces incommodités et améliorer substantiellement la qualité du riz étuvé, un dispositif d'étuvage amélioré a été développé par la recherche en collaboration avec les femmes étuveuses du riz. Ce dispositif est composé d'une marmite en fonte d'aluminium et d'un bac d'étuvage en tôle galvanisé perforé à la base pour faciliter la précuisson à la vapeur. L'équipement peut être fabriqué par des artisans locaux avec les matériaux et les compétences disponibles. Le principe derrière cette technologie améliorée est qu'après avoir trempé le paddy, il est transféré dans le récipient supérieur et précuit à la vapeur, sans que le paddy ne touche l'eau (Zossou et al., 2009). Malgré le succès que ce dispositif amélioré d'étuvage du riz a eu auprès de nombreuses femmes étuveuses du riz au Bénin (Zossou et al., 2012), son utilisation ne permet pas l'étuvage de grandes quantités de riz. Pour pallier cette limite, la recherche en collaboration avec les femmes étuveuses a développé le système amélioré d'étuvage du riz dénommé «GEM ». Il est espéré de ce dernier des changements technicoorganisationnels précurseurs d'une amélioration de la qualité et de la quantité du riz étuvé au niveau local. Le processus d'étuvage généré par la méthode «GEM » s'est résumé dans le tableau 2: 
Tableau 2: Description des étapes améliorées d'étuvage du riz avec le GEM (Description of the improved stages for steaming rice with GEM)

\begin{tabular}{cl}
\hline \multicolumn{1}{c}{ Etapes } & \multicolumn{1}{c}{ Description des étapes } \\
\hline $\begin{array}{c}\text { Vannage et le } \\
\text { triage }\end{array}$ & $\begin{array}{l}\text { Le vannage et le triage se font de manière consécutive. Ils consistent à vanner le paddy } \\
\text { à l'aide de vannes et à trier à main les bons grains des mauvais. Le vannage et le triage } \\
\text { permettent de débarrasser les grains de ses impuretés (sable, débris et pailles...) et grains } \\
\text { non mûrs. }\end{array}$ \\
\hline
\end{tabular}

Triple lavage Le paddy est lavé trois (3) fois successivement dans trois (3) bassines contenant une grande quantité d'eau. Ce triple lavage permet de débarrasser le paddy des impuretés (grains de sable, résidus de récolte etc.) et des grains non mûrs. Ces impuretés et grains non mûrs qui surnagent lors du lavage sont collectés à l'aide d'un petit panier ou d'une passoire. Le sable qui se dépose au fond de la bassine est jeté après avoir récupéré minutieusement le paddy lavé.

1er égouttage Le paddy lavé est versé dans un égouttoir en acier pour faire égoutter l'eau.

Trempage à Après l'égouttage, le paddy est versé dans le kit de trempage. Parallèlement, un certain

l'eau chaude volume d'eau est chauffé dans un bac d'étuvage en acier. A l'aide d'un foyer amélioré, l'eau est portée à l'ébullition. Grâce à la pompe à eau chaude, l'eau bouillante est déversée dans le kit de trempage par un tuyau. Cette opération vise l'élévation de la température. Après le chauffage, le paddy est laissé jusqu'au lendemain soit $12 \mathrm{~h}$ de temps environ pour se refroidir.

\begin{tabular}{cl}
$\begin{array}{c}\text { 2ème } \\
\text { égouttage }\end{array}$ & $\begin{array}{l}\text { Le lendemain, le paddy contenu dans le kit de trempage est vidé de son eau grâce à un } \\
\text { bouchon réalisé en bas de ce kit. }\end{array}$ \\
\hline $\begin{array}{c}\text { Pré-cuisson à } \\
\text { la vapeur }\end{array}$ & $\begin{array}{l}\text { Après l'égouttage, le paddy toujours dans l'égouttoir est inséré jusqu'à un certain niveau } \\
\text { dans un bac d'étuvage contenant de l'eau propre. Cette quantité d'eau ne doit pas toucher } \\
\text { le fond de l'égouttoir pour éviter que le paddy soit mouillé. L'eau est portée à l'ébullition } \\
\text { au niveau du foyer amélioré. La vapeur de l'eau bouillante produit de la chaleur qui pré- } \\
\text { cuit le paddy contenu dans l'égouttoir. Ce qui évite les grains du riz brûlés. }\end{array}$ \\
\hline Séchage & $\begin{array}{l}\text { Le paddy pré-cuit est séché au soleil sur une aire de séchage. La fin du séchage conduit } \\
\text { au décorticage et stockage. }\end{array}$ \\
\hline Décorticage & $\begin{array}{l}\text { Il se fait avec une décortiqueuse moderne octroyée par AfricaRice aux femmes } \\
\text { étuveuses. Ce matériel permet de réduire le taux de brisures du riz paddy. }\end{array}$ \\
\hline Stockage & Le stockage se fait dans des sacs de $5 \mathrm{~kg}, 10 \mathrm{~kg}$ ainsi que dans des sacs de $50 \mathrm{~kg}$. \\
\hline
\end{tabular}

Source : Données de terrain

\section{Génération d'innovations technologiques au sein de la PI}

L'usage du système amélioré d'étuvage du riz «GEM» a significativement amélioré les pratiques des femmes en matière d'étuvage du riz et ce, à toutes les étapes du processus (Tableau 3). En effet, du vannage jusqu'au stockage en passant par les autres étapes intermédiaires, le test de Wilcoxon montre une avancée significative en matière d'améliorations apportées par le nouveau système d'étuvage «GEM ». Cette amélioration est due au fait que le nouveau système «GEM» intègre des étapes supplémentaires qui pallient les insuffisances des systèmes antérieurs ${ }^{1}$. Il s'agit notamment du vannage, du triage et du triple lavage du riz paddy ; ce qui permet d'éliminer la quasi-totalité des impuretés dès le début du processus

${ }^{1}$ Il s'agit du système traditionnel et du système semi-moderne d'étuvage du riz 
d'étuvage. En outre, la pré-cuisson à la vapeur, préconisée par «GEM », permet une homogénéité des grains après cette étape ; contrairement à la méthode traditionnelle qui fait usage d'eau pour la pré-cuisson avec pour corollaire des grains hétérogènes à la fin du processus. Par ailleurs, le système «GEM » a-t-il apporté des améliorations aux étapes pré-existantes afin de garantir une meilleure qualité du riz à la fin du processus d'étuvage.

En plus du changement collectif engendré par «GEM» en termes de pratiques d'étuvage dans les groupements ; 99,67\% des femmes ont affirmé avoir amélioré leurs pratiques individuels en s'inspirant des améliorations collectives liées à l'usage de ce nouveau système.

Par ailleurs, le système amélioré «GEM» a-t-il permis une amélioration hautement significative ${ }^{2}$ de la quantité de riz étuvée en une session d'étuvage qui dure deux jours. En effet, toute chose étant égale par ailleurs, le système traditionnel permet d'étuver $193 \mathrm{~kg}$ de riz paddy en une session d'étuvage, alors que le système amélioré « GEM » porte cette quantité à $356 \mathrm{~kg}$.

En somme, la Plateforme d'Innovation a permis à travers le système amélioré «GEM », des avancées remarquables tant sur la qualité ${ }^{3}$ que sur la quantité du riz étuvé. La pénibilité du travail a été également allégée.

Tableau 3 : Test de Wilcoxon à deux échantillons dépendants (score après GEM-score avant GEM)

\begin{tabular}{|c|c|c|c|c|c|c|c|}
\hline Désignations & Triage & Lavage & Trempage & $\begin{array}{l}\text { Pré- } \\
\text { cuisson }\end{array}$ & Séchage & Décorticage & Stockage \\
\hline Après GEM & 1,855 & 2,607 & 2,702 & 2,842 & 2,562 & 2,977 & 2,193 \\
\hline Avant GEM & 0,247 & 0,43 & 2,608 & 2,76 & 2,487 & 2,907 & 2,157 \\
\hline $\begin{array}{c}\text { Diff (Après GEM } \\
\text { - Avant GEM) }\end{array}$ & $+1,608 * * *$ & $+2,177 * * *$ & $+0,094 * * *$ & $+0,082 * * *$ & $+0,075 * * *$ & $+0,07 * *$ & $+0,036 * * *$ \\
\hline
\end{tabular}

Source : Données de terrain

\section{Génération d'innovations organisationnelles}

Le système amélioré « GEM », à travers la Plateforme d'Innovation a engendré des mutations positives entre les femmes étuveuses d'une part et entre les groupes d'acteurs de la Plateforme d'Innovation d'autre part.

Pour ce qui concerne les femmes étuveuses, l'on note une structuration de ces dernières en groupements, avec à leur tête un bureau directeur. Ce bureau, composé de trois membres assure le bon fonctionnement du groupement et

\footnotetext{
${ }^{2}$ Seuil de $1 \%$ (test $\mathrm{t}$ de Student)

${ }^{3}$ On note désormais un faible taux de brisure, une diminution considérable des impuretés et des grains du riz brûlés avec le système amélioré « GEM ».
} 
représente ses membres partout où nécessité y est. Il convient de signaler que les prises de décisions au sein des groupements sont participatives afin de garantir la transparence et l'intérêt commun. Une telle inclusion a eu des effets positifs sur les relations qu'entretiennent les femmes étuveuses avec leurs pairs. En effet, 91,33\% d'entre elles, ont affirmé avoir connu une amélioration des relations avec les autres membres du groupe. En outre, $89 \%$ ont pu noter une bonne ambiance entre les responsables de leur groupe. Par ailleurs, $98,67 \%$ ont désormais confiance à leurs responsables et sont suffisamment satisfaites de leur gestion.

Quant aux relations entre groupes d'acteurs de la Plateforme d'Innovation, la figure 2 montre que la quasi-totalité des femmes étuveuses avait de faible relation avec les institutions comme les IMF (99,67\% de femmes), le SCDA (96\%), l'INRAB (99\%) et les producteurs de riz (93\%). Par contre, après l'introduction du système «GEM », la tendance s'est fortement renversée. On note désormais une amélioration des relations entre les femmes et les institutions supra-évoquées (figure 2).

En effet, 95\% des femmes bénéficient désormais, facilement des services financiers à la Caisse Locale de Crédit Agricole Mutuel (CLCAM) contre $0,33 \%$ par le passé. En outre, l'amélioration des relations entre les femmes et les SCDA/INRAB se traduit par l'effort supplémentaire fourni par ces institutions en vue d'assurer un meilleur accompagnement aux acteurs de la chaine de valeur du riz en occurrence les producteurs qui fournissent du paddy aux femmes étuveuses.

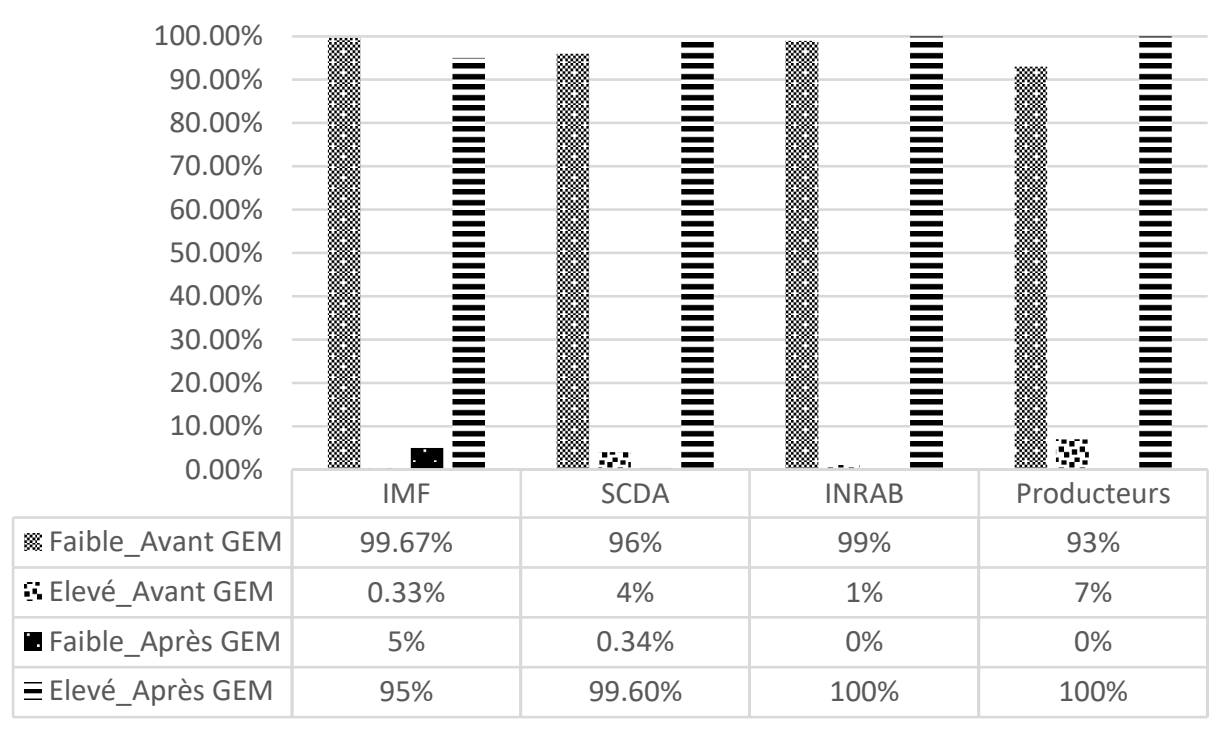

Figure 1: Niveau de relation entre les femmes étuveuses et IMF, SCDA, INRAB, producteurs

Source : Données de terrain 


\section{Diffusion d'innovations}

Il ressort de l'étude que $76,33 \%$ des femmes ont diffusé des informations relatives au «GEM » à travers l'effet tache d'huile. Ainsi, une femme étuveuse ayant participé aux formations pratiques sur le système d'étuvage « GEM », a touché en moyenne 9 autres femmes étuveuses dans son voisinage proche ou lointain.

Aussi, est-il clairement démontré que plus l'innovation est adaptée à la situation réelle des femmes étuveuses, plus rapide sera sa diffusion.

Afin de comprendre les facteurs qui influencent la diffusion du système amélioré «GEM », nous avons eu recours au modèle Logit binomial dont les résultats sont consignés dans le tableau 4.

Les résultats indiquent un ratio de vraisemblance égal à 23,19. Cette valeur est supérieure à celle de $\chi^{2}$ théorique égale à 14,07 à cinq degrés de libertés (ddl), au seuil de $1 \%$. Le modèle est donc globalement significatif au seuil de $1 \%$. Toutefois, il est nécessaire de préciser avant toute interprétation que dans un modèle binaire (Logit dans le cas de la présente étude), que les paramètres des variables du modèle Logit ne sont pas directement interprétables. En effet, le plus important reste le signe des coefficients qui indique si la variable associée influe sur la probabilité à la hausse ou à la baisse (Doucouré 2005).

Ainsi, les résultats de l'estimation des coefficients révèlent que l'âge, le statut de membre de bureau d'un groupement et le niveau d'instruction augmentent les chances de diffuser la technologie «GEM ». Par contre, les autres variables telles que la taille du ménage et le niveau d'expérience n'ont aucune influence significative sur la diffusion de cette technologie.

Comme signaler plus haut, la valeur numérique des coefficients du modèle Logit ne propose pas des interprétations directes. A ce titre, les effets marginaux ont été utilisés pour avoir une idée des niveaux d'influence.

Ainsi, l'effet marginal associé à la variable « âge » est de 0,29 environ. Ce qui signifie que : plus l'âge du répondant augmente, la probabilité de diffuser la technologie «GEM » augmente de $29 \%$.

Le niveau d'instruction quant à lui, présente un effet marginal de 0,02. Ainsi, plus la femme étuveuse est instruite, la probabilité qu'elle diffuse l'innovation « GEM » augmente de $2 \%$.

Enfin, le statut de membre de bureau d'un groupement augmente de $11 \%$ la probabilité de diffuser la technologie «GEM ». Cette variable présente un effet marginal de 0,11 . 
Tableau 4: Résultats de la régression logistique binaire sur les facteurs déterminant la diffusion du GEM.

\begin{tabular}{|c|c|c|}
\hline \multicolumn{3}{|c|}{ Méthode d'estimation: Maximum de vraisemblance (modèle logit binaire) } \\
\hline Variables & Coefficients & Effets marginaux \\
\hline Membre de bureau & $1,421 *$ & $0,107^{*}$ \\
\hline Age & $1,926 * *$ & $0,292 * *$ \\
\hline Taille du ménage & 0,420 & 0,031 \\
\hline Niveau d'instruction & $0,286 * *$ & $0,021 * *$ \\
\hline Niveau d'expérience & 0,062 & 0,005 \\
\hline Constante & $-0,239$ & - \\
\hline Pseudo R2 de McFadden & \multicolumn{2}{|c|}{0,116} \\
\hline Statistique LR $(\mathrm{ddl}=5)$ & \multicolumn{2}{|c|}{$23,19 * * *$} \\
\hline Log Likelihood & \multicolumn{2}{|c|}{$-88,108$} \\
\hline
\end{tabular}

* Seuil de significativité à $1 \%$, ** Seuil de significativité à $5 \%$, *** Seuil de significativité à $10 \%$.

Source : Données de terrain

\section{Discussions}

Les résultats ont montré que la mise en place d'une technologie innovante comme le système amélioré «GEM » a servi de catalyseur pour la dynamisation de la plateforme d'innovation par le renforcement de l'apprentissage, des interactions et des institutions. La mise en place de cette innovation a eu des effets positifs tant sur le plan organisationnel que sur la qualité du produit fini qui est le riz étuvé. En effet, à travers cette plateforme et le système amélioré «GEM », les femmes étuveuses ont pu être regroupées et leurs pratiques d'étuvage ont connu beaucoup d'améliorations. Ces résultats corroborent les travaux d'Adekunle et al (2012) et de Gouwy (2018). Selon ces auteurs, une plateforme dynamique dans le processus de production d'innovation est un atout pour faciliter les interactions tout en renforçant l'apprentissage et les institutions. Ce qui renforce le caractère multidimensionnel et processuel de l'innovation agricole relevé par Shut al., 2016 et Zossou et al., 2020.

La collaboration entre les groupes d'acteurs engagés dans le processus de production d'innovation dans la chaine de valeur du riz étuvé a en effet facilité l'accès des femmes étuveuse de riz aux crédits auprès des institutions formelles de microfinance. Ce résultat confirme celui de Sidibé et al. (2017) qui affirme que l'une des priorités d'une PI est de faciliter des échanges ouverts entre les organisations des professionnels agricoles et les organismes financiers. 
En dehors des services financiers, les résultats ont également montré une forte amélioration des relations avec les autres acteurs que sont : les producteurs du riz, les structures d'encadrement agricole et les centres de recherche. Ces structures pour la plupart, ont contribué au renforcement des capacités des femmes étuveuses à travers des formations théoriques et pratiques. Ces résultats vont dans le même sens que ceux de Gouwy (2018) qui ont montré que la plateforme d'innovation est un mécanisme visant à renforcer les capacités de communication, d'interaction et d'innovation entre les acteurs mutuellement dépendants dans une chaîne de valeur agricole.

S'agissant des facteurs pouvant influencer la diffusion de l'innovation «GEM », il a été noté des facteurs intrinsèques aux femmes étuveuses et des facteurs liés au contexte socio-économique et culturel tels que l'âge, le niveau d'instruction et l'appartenance à un bureau dirigeant les groupements de femmes. Ces facteurs font partie intégrante de ceux mis en exergue par Rogers (1999) ; Meijer et al. (2015) ; et Shikuku (2019) dans les théories d'adoption et de diffusion des innovations agricoles. L'influence des leaders de groupements de producteurs sur la diffusion des innovation agricoles a été également mise en évidence par Van Eck et al. (2011). De même, l'influence du niveau d'instruction et de l'âge sur la diffusion des innovations agricoles a été mise en évidence par Issoufou et al. (2017).

\section{Conclusion et Implications}

La post-récolte du riz fait face à d'énormes défis tels que la diversité des acteurs et de leurs intérêts, l'insuffisance d'appui technique, la mauvaise qualité du produit final conduisant à sa faible compétitivité sur le marché national et international, la politique locale non adéquate aux exigences du marché international et les faiblesses sur le plan institutionnel. La plateforme d'innovation est perçue donc comme une approche multidisciplinaire et multiacteurs qui pourra efficacement et effectivement explorer les stratégies pour relever les nombreux défis qui s'imposent au développement de la chaîne de valeur de riz au Bénin. Cependant la dynamique, le bon fonctionnement, le progrès et le succès d'une plateforme d'innovation dépendent du climat de confiance entre les membres, de leur volonté á travailler ensemble et l'existence d'initiatives pour servir de catalyseur.

L'introduction dans la plateforme d'innovation de la technologie améliorée d'étuvage de riz a servi de catalyseur pour une meilleure interaction entre les parties prenantes. Ce qui a conduit au renforcement de l'apprentissage individuel et social ; à la génération d'innovations et à leur diffusion pour une adoption et ou adaptation. Les changements notés dans les pratiques des acteurs à la base de la PI concernent principalement l'introduction de nouvelles étapes dans le processus d'étuvage telles que le vannage, le triage, le triple lavage et la pré-cuisson du paddy à la vapeur. Les 
changements technologiques ont été accompagnés d'une meilleure organisation des femmes étuveuses en associations. Ce renforcement sur le plan organisationnel a facilité l'accès aux institutions financières décentralisées formelles et informelle et une diffusion des innovations générées.

L'originalité de la présente étude est l'utilisation d'une perspective systémique pour comprendre le processus de génération et de diffusion d'innovation relative à la post-récolte du riz local dans un contexte agricole marquée par un faible taux d'alphabétisation, un taux élevé de ruralité et la prédominance de petits exploitants agricoles. Cette étude confirme les approches systémiques multi-acteurs sont propices au contexte sub-saharien marqué par une diversité des acteurs agricoles et de leurs intérêts. L'utilisation de ces approches dans un tel contexte favorise le développement et l'amélioration des chaines de valeurs agricoles grâce aux actions combinées d'une multitude de parties prenantes interconnectées et en synergie d'actions.

\section{References:}

1. Adekunle, A.A., Fatunbi, A.O. (2012). Approaches for setting-up multi-stakeholder plateforms for agricultural research and development. World applied sciences journal 16 (7) : 981-988.

2. Ahoyo, A.N.R. (2006). Monographie de la commune de Malanville. Cotonou, Bénin.

3. Angbo-Kouakou, E., Temple, L., Mathé, S. et Assemien, A. (2016). Plateformes d'innovation comme dispositif d'orientation des trajectoires technologiques des filières agricoles. Cas de la filière banane plantain en Côte d'Ivoire. ISTE Ltd., UK-openscience.fr, London, UK.

4. CORAF. (2011). Analyse des mécanismes de diffusion des technologies agricoles améliorées et innovations dans l'espace CEDEAO. Dakar, Sénégal.

5. Faure, G., Toillier, A., Havard, M., Rebuffel, P., Moumouni, I.M., Gasselin, P., et Tallon, H. (2018). Le conseil aux exploitations agricoles pour faciliter l'innovation : Entre encadrement et accompagnement. 11èmé chapitre d'innovation et développement dans les systèmes agricoles et alimentaires. Editions Quæ. Versailles, Cedex.

6. Houssou, F.A.P., Ahoyo Adjovi, R.N., Dansou, V., Hounyevou-Klotoe, A., Hotegni, B.A. et Mensah, A.G. (2016). Guide pratique de production du riz blanc au Bénin : aspect post-récolte. Programme Technologiques Agricole et Alimentaire, Porto-Novo, Bénin.

7. ILRI. (2013). Directives pour les Plateformes d'Innovation: Facilitation, Suivi et Évaluation. CGIAR, Addis Abeba, Ethiopia. 
8. INRAB. (2014). Rapport d'atelier d'initiation des Plateformes MultiActeurs et de planification des activités dans les pôles de développement rizicole de Glazoué et de Bantè. INRAB, Cotonou, Bénin.

9. INSAE. (2013). RGPH4 : Que retenir des effectifs de population en 2013 ? Cotonou, Bénin.

10. Issoufou, O.H., Boubacar, S., Adam, T. et Yamba, B. (2017). Déterminants de l'adoption et impact des variétés améliorées sur la productivité du mil au Niger. African Crop Science Journal 25(2) : 207-220.

11. Jeunier, B., Long, J.S., et Brandibas, G. (2000). L'approche systémique en sciences humaines : et les données dans tout ça? Première partie: aspects théoriques. Les dossiers des sciences de l'education 200(3) : 143-159.

12. Konnon, D.D., Sotondji, C. et Adidéhou, A. (2014). Etat des lieux de la filière riz au Bénin en 2014. Rapport d'étude: Conseil de concertation des riziculteurs du Bénin. Disponible sur www.interreseaux.org/IMG/pdf/rapport_Final_Etat_des_Lieux_Riz 1.pdf.

13. Lawin, K.G. (2006). Analyse des déterminants de l'adoption et de la diffusion du dispositif amélioré d'étuvage du riz dans la commune de Glazoué. Mémoire d'ingénieur agronome à la FSA/UAC, AbomeyCalavi, Bénin.

14. Leeuwis, C. (2010). Changing views of agricultural innovation: implications for communicative intervention and science. Research to impact: case studies for natural resource management for irrigated rice in Asia. In Palis, F.G., Singleton, G.R., Casimero, M.C., and Hardy, B. (eds.), Research to impact: case studies for natural resource management for irrigated rice in Asia. IRRI, Manila, Philippines, pp. 15-32.

15. Gouwy, L. (2018). Le concept des plateformes d'innovation en agriculture: analyse bibliographiques et illustrations avec deux exemples agroforestiers sur l'hévéa en Thaïlande et sur le café au Nicaragua. CIRAD et AgroParisTech, France.

16. MAEP. (2007). Livre blanc sur le conseil agricole au Bénin : Projet d'Appui Institutionnel à la Modernisation de l'Agriculture Familiale. MAEP, Cotonou, Bénin.

17. Meijer, S. S., D. Catacutan, O. C. Ajayi, G. W. Sileshi, and M. Nieuwenhuis. 2015. The Role of Knowledge, Attitudes and Perceptions in the Uptake of Agricultural and Agroforestry Innovations among Smallholder Farmers in Sub-Saharan Africa. International Journal of Agricultural Sustainability 13 (1): 40-54. 
18. Nederlof, S. et Pyburn, R. (2012). One finger connot lift a rock: Facilitating innovation platforms to trigger institutional change in West Africa. Kit publishers, Amsterdam.

19. Pouch, T. (2015). L'agriculture et l'enjeu de l'innovation : dimensions générales et éclairage méditerranéen. Université de Reims Champagne Ardenne, Paris.

20. Rogers, E. M. 1995. Diffusion of Innovations. 4th ed. New York: Free Press.

21. Schut, M., Klerkx, L., Sartas, M., Lamers, D., Mc Campbell, M., Ogbonna, I., Kaushik, P., Atta-Krah, K. and Leeuwis, C. (2016). Innovation platforms: experiences with their institutional embedding in agricultural research for development. Experimental Agriculture $52: 537-561$.

22. Shikuku, K. M. 2019. Information Exchange Links, Knowledge Exposure, and Adoption of

23. Agricultural Technologies in Northern Uganda. World Development 115: 94-106.

24. Sidibé, A., Vellema, S., Dembelé, F., Traoré, M. et Kuyper, W.T. (2017). Analyse d'une plate-forme d'innovation dans la filière karité au Mali. Cah. Agric. 26(4): 1-8 .

25. Homann-Kee Tui, S., Adekunle, A., Lundy, M., Tucker, J., Birachi, E., Schut, M., Klerkx, L., Ballantyne, P., Duncan, A., Cadilhon, J. et Mundy, P. (2013). What are innovation platforms? ILRI Technical Report, Innovation platforms practice brief 1: 1-6.

26. Van Eck, P.S., Jager, W. et Leefland, P.S.H. (2011). Opinion leader's role in innovation diffusion: A simulation study. Journal of Product Innovation Management. 28(2) : 187-203.

27. Van Mele, P., Wanvoeke, J. and Zossou, E. (2010). Enhancing rural learning, linkages and institutions: the rice videos in Africa. Development in Practice 20 (3) : 414-421.

28. Zossou, E. (2013). Etude d'impact de l'apprentissage par la vidéo et la radio rurale sur la transformation à petite échelle du riz local au Bénin. Thèse de doctorat, Université de Liège -Gembloux Agro-Bio Tech.

29. Zossou, E., Arouna, A., Diagne, A. et Agboh-Noameshie, A.R. (2020). Learning agriculture in rural areas: the drivers of knowledge acquisition and farming practices by rice farmers in West Africa. The Journal of Agricultural Education and Extension 26 (3): 291-306.

30. Zossou, E., Van-Mele P., Wanvoeke, J. \& Lebailly, Ph. (2012). Participatory impact assessment parboiling videos with women in Benin. Experimental Agriculture. 48 (3): 438-447. 
31. Zossou, E., Van-Mele, P., Vodouhe, S.D. et Wanvoeke, J. (2009). The Power of Video to Trigger Innovation: Rice Processing in Central Benin. International Journal of Agricultural Sustainability 7 (2): 119129. 